cells - insulinoma-1 (INS-1) cells that respond to glutamate application with a rapid elevation in their intracellular calcium concentration owing to the expression of NMDA receptors. The authors showed that the flashes of fluorescence at the astrocyte membrane were followed by an NMDA receptor-dependent increase in intracellular calcium in the INS-1 cells, confirming that glutamate was being released through regulated exocytosis.

Although the astrocytic glutamate-releasing vesicles are similar to synaptic vesicles in many ways, there are also some intriguing differences. For example, the groups of vesicles were often smaller and less well-organized than the vesicles at presynaptic terminals. Also, they express the v-SNARE cellubrevin, whereas synaptic vesicles mostly express VAMP2 (vesicle-associated membrane protein 2). In the future, it will be interesting to explore the implications of these similarities and differences for neuron-glia communication.

Heather Wood

\section{(2) References and links}

original RESEARCH PAPER Bezzi, P. et al. Astrocytes contain a vesicular compartment that is competent for regulated exocytosis of glutamate. Nature Neurosci. 7, 613-620 (2004)

FURTHER READING Haydon, P. G. Glia: listening and talking to the synapse. Nature Rev. Neurosci. 2, 185-193 (2001) |

Newman, E. A. New roles for astrocytes: regulation of synaptic transmission. Trends Neurosci. 26, 536-542 (2003)

those that contain NR2B. The idea is also consistent with previous findings, such as the fact that the NR2A/NR2B ratio increases during development, and LTD is more difficult to induce in more mature animals. However, there are also some problems for the theory. For example, a previous study by Tang et al. found that overexpression of NR2B subunits in the forebrain of mice increased levels of LTP, not LTD.

Furthermore, different rules might apply in different parts of the brain.

Further work will no doubt help to clarify such issues, and should establish how different stimulation protocols might preferentially activate receptors that contain different types of subunit. It should also investigate what might happen downstream of receptor activation: there is already evidence that NR2A-containing receptors might couple to different signalling pathways from NR2Bcontaining receptors, and this provides a potential mechanism for their different effects on long-term plasticity.

(9)) References and links Rachel Jones ORIGINAL RESEARCH PAPER Liu, L. et al. Role of NMDA receptor subtypes in governing the direction of hippocampal synaptic plasticity. Science 304, 1021-1024 (2004) FURTHER READING Tang, Y. P. et al. Genetic enhancement of learning and memory in mice. Nature 401, 63-69 (1999)

\title{
ION CHANNELS
}

\section{Crystal-clear interactions}

Calcium channels are the latest family to give in to the power of crystallography and reveal their structural details. Three recent papers have disclosed the atomic structure of the interacting domains between $\alpha$ - and $\beta$-subunits of voltagegated calcium channels, challenging some previous ideas about the workings of the channel.

Voltage-gated calcium channels comprise different subunits - the $\alpha_{1}$-subunit forms the pore of the channel, and the $\alpha_{2}-, \beta$ - and $\gamma$-subunits modulate channel function. The $\beta$-subunit, in particular, has a profound effect on several channel properties, including activation and inactivation rates and surface expression. Previous studies had narrowed down the $\alpha_{1}$ - and $\beta$-regions that mediate their interaction — the so-called $\alpha_{1}$-interaction domain (AID) and a conserved core of the $\beta$-subunit, which includes what was regarded as the $\beta$-interaction domain (BID). The three papers report on the crystal structure of the conserved core of several $\beta$-subunits on their own and bound to the AID.

Perhaps the most surprising result from the three papers is that the BID does not really interact with the $\alpha$-subunit. Instead, it is largely buried within the $\beta$-core and is more relevant for the structural integrity of the actual binding site, which, as it turns out, is structurally reminiscent of another protein family - membraneassociated guanylate kinases (MAGUKs).

MAGUKs are known to function as scaffolds at the synapse, a function for which their several PDZ domains are critical. The PDZ domains, which are absent in the core of the $\beta$-subunit, precede an $\mathrm{SH} 3$ domain and a guanylate kinase domain. A linker domain joins these two regions and, together, these three domains constitute the region of homology between MAGUKs and the conserved $\beta$-core. The structural analysis showed that the domain of the $\beta$-subunit that binds the AID is a hydrophobic cleft within the region homologous to the guanylate kinase domain of MAGUKs; the BID, by contrast, lies in the linker domain.

From the functional perspective, the structural results provide good clues about the way in which the $\beta$-subunit can affect channel inactivation. The interaction between the two subunits places the $\beta$-region near the intracellular end of a pore-lining segment of the $\alpha$-subunit that is important for switching the channel off, raising the possibility that the $\beta$-subunit can affect the movement of this segment through electrostatic or physical interactions.

Last, the AID seems to occupy a small part of the region of homology between MAGUKs and the $\beta$-subunit. As this region contains other protein-protein interaction modules, such as the $\mathrm{SH} 3$ domain, it is conceivable that additional molecules bind to this region, potentially increasing the complexity of calcium channel modulation.

Juan Carlos López, Chief Editor, Nature Medicine

\section{(2) References and links}

ORIGINAL RESEARCH PAPERS Van Petegem, F. et al. Structure of a complex between a voltage-gated calcium channel $\beta$-subunit and an $\alpha$-subunit domain. Nature 429, 671-675 (2004) | Opatowsky, Y. et al. Structural analysis of the voltage-dependent calcium channel $\beta$ subunit functional core and its complex with the $\alpha_{1}$ interaction domain. Neuron 42, 387-399 (2004) | Chen, Y. et al. Structural basis of the $\alpha-\beta$ subunit interaction of voltage-gated $\mathrm{Ca}^{2+}$ channels. Nature 429, 675-680 (2004) 